\title{
Hauptergebnisse und Erfahrungen durchgeführter Innovations- und Technikanalysen zu Akzeptanz und Nachfrage auf den Gebieten Pflanzengentechnik und seniorengerechte Technik
}

\author{
Martina Brandt, Ulla Große, Frank Hartmann, Rainer Voß
}

\section{Das Konzept Innovations- und Technikanalyse}

Anknüpfend an langjährige Erfahrungen aus der „klassischen" Technikfolgenabschätzung (TA) entwickelte das BMBF im Jahre 2000 das Konzept der Innovations- und Technikanalyse (ITA). Mit dem Ziel, Gestaltungs- und Handlungsoptionen bezogen auf Technikentwicklung und Innovationsprozesse zu erarbeiten, orientiert ITA auf die Analyse der gesellschaftlichen Anwendungsbedingungen und möglichen Folgen neuer Technologien in Frühphasen ihrer Entwicklung. So sollen bereits im Stadium der Erarbeitung von Leitvisionen und Szenarien der Forschungsförderung durch das BMBF innovationsorientierte Bewertungen der Voraussetzungen und Folgen von Technikentwicklung und -anwendung vorgenommen werden. [1]

Innovations- und Technikanalysen haben nicht schlechthin Technologien zum Untersuchungsgegenstand, sondern wissenschaftlich-technische und organisatorische Innovationen. Damit richtet sich der Blick verstärkt auch auf die Wirtschaft als jenen Bereich, in dem Innovationen maßgeblich generiert und entwickelt werden und ihre Anwendung finden. Die Einbeziehung der Wirtschaft und ihrer Instrumente der Produktfolgenabschätzung soll zu Synergien mit einer integrierten, modernen Innovations- und Technikanalyse fuihren. [2]

Beibehalten wird die Sichtweise „klassischer“ TA, dass die Politik ein wesentliches gesellschaftliches Subsystem darstellt, das durch die Gestaltung von Rahmenbedingungen Technikentwicklung beeinflusst und somit ein wichtiger Adressat für TA ist.

Die Innovationsorientierung von TA bedeutet nicht zwangsläufig eine Schwerpunktverlagerung auf einzelne Unternehmen, denn die Hervorbringung und Realisierung von Innovationsprozessen erfolgt i. d. R. nicht innerbetrieblich, sondern zwischenbetrieblich und interinstitutionell und es werden auch private Endverbraucher frühzeitig in den Innovationsprozess eingebunden. Insofern geht es ITA um die Berücksichtigung übergreifender Aspekte der Gestaltung vielfältiger Ressourcenaustauschprozesse zwischen Unternehmen und ihren Umwelten in Innovationsprozessen. Zum Adressatenkreis zählen folglich neben politischen Akteuren auch Unternehmen und Konsumenten.

Darüber hinaus unterstellt das ITA-Konzept, dass Bürger und die Öffentlichkeit bestimmte Einstellungen zur Tech- nik haben und dementsprechend Technikentwicklung blockieren, hemmen oder fördern können. Folglich liegt ein weiterer Schwerpunkt von ITA auf der Untersuchung von Einflussfaktoren auf solche Einstellungen sowie der Einbeziehung der Öffentlichkeit in die Diskussion um die Entwicklung neuer Technologien. Über rein analytische Fragen hinaus gewinnen in diesem Prozess diskursive und partizipative Aspekte an Bedeutung.

Diese Perspektivenerweiterungen und -vertiefungen im Vergleich zu „klassischer“ TA fanden im Jahre 2000 ihren ersten umsetzungsbezogenen Niederschlag in den Ausschreibungen des BMBF zu den Themenfeldern „Technikakzeptanz und Nachfragemuster als Standortvorteil“, „Evaluation internetgestuitzter Diskurse“ und „Formen und Reichweite elektronischer Demokratie“.

Aufgrund Ihrer langjährigen Erfahrungen mit innovationsorientierter Technikfolgenabschätzung beteiligte sich die Technische Fachhochschule in Kooperation mit dem Institut für Regionale Innovationsforschung an der Vertiefung und Umsetzung des ITA-Konzepts durch die Bearbeitung von zwei Projekten im Themenfeld „Technikakzeptanz und Nachfragemuster als Standortvorteil“", die beide im Jahre 2002 erfolgreich abgeschlossen wurden: ${ }^{1}$

- Technikakzeptanz und Nachfragemuster als Standortvorteil im Bereich der Pflanzengentechnik

- Determinanten der Technikaufgeschlossenheit und des Nachfrageverhaltens in Bezug auf seniorengerechte Technik

Beide Projekte weisen sowohl konzeptionelle Gemeinsamkeiten als auch Unterschiede auf. Im nachfolgenden Abschnitt wird vergleichend gezeigt, wie die o. g. Aspekte des ITA-Konzepts aufgegriffen, konzeptualisiert und empirisch untersucht wurden. Um die Lesbarkeit des Textes zu erleichtern, werden die Projekte im Weiteren verkürzt als „Pflanzengentechnik-Projekt“ sowie als „Senioren-Projekt" bezeichnet.

\section{Projektansätze und Methoden im Vergleich}

In beiden Projekten wird davon ausgegangen, dass die wirtschaftliche Umsetzung des Potenzials neuer Technologien durch Innovationsprozesse erfolgt, was die Kombination mit bestehenden und/oder neuen Technologien beinhaltet. Unterschiedlich sind jedoch die untersuchten 
Technologiefelder und Innovationsprozesse. Im Bereich der Pflanzengentechnik kann man von einer einheitlichen technologischen Basis mit einer Vielzahl von Anwendungsrichtungen ausgehen, im Bereich seniorengerechte Technik von unterschiedlichen Technologien und unterschiedlichen Anwendungen. Letztere wurden in den Bereichen Mobilität, Sicherheit, Kommunikation und Haushalt/Wohnen untersucht, tangieren ein äußerst vielfältiges Technologiespektrum und betreffen Produkte unterschiedlichen Technologieniveaus (vgl. Abbildung 1). In beiden Projekten wird davon ausgegangen, dass Innovationsprozesse in Wertschöpfungsketten realisiert werden. Diese unterscheiden sich je nach Anwendungsfeld hinsichtlich der Stufen und der beteiligten Innovationsakteure. Die Vertikalität und die Stufigkeit der Wertschöpfungsketten sind bei der Pflanzengentechnik besonders ausgeprägt (vgl. Abbildung 2).
Den Projekten liegt ein Innovationsverständnis zugrunde, demzufolge die Innovationsdynamik aus den Zusammenhängen zwischen technikerzeugenden, -verwendenden und regulierenden Sozialsystemen resultiert, die durch entsprechende Akteure gestaltet werden. [3] Innovationen werden nicht in einem linear verlaufenden Prozess generiert, sondern entstehen vielmehr in einem mehrstufigen Prozess, der durch rekursiv verknüpfte Kommunikationen zwischen Technikerzeugern und Technikverwendern gekennzeichnet ist. Dieser vollzieht sich unter Randbedingungen, die insbesondere vom technikregulierenden Sozialsystem gesetzt sind und den Prozess konditionieren.

Aufgrund der wirtschaftsbezogenen Perspektive von ITA, die sich im genannten Themenfeld insbesondere mit dem Begriff der Nachfrage verbindet, erreichen beide
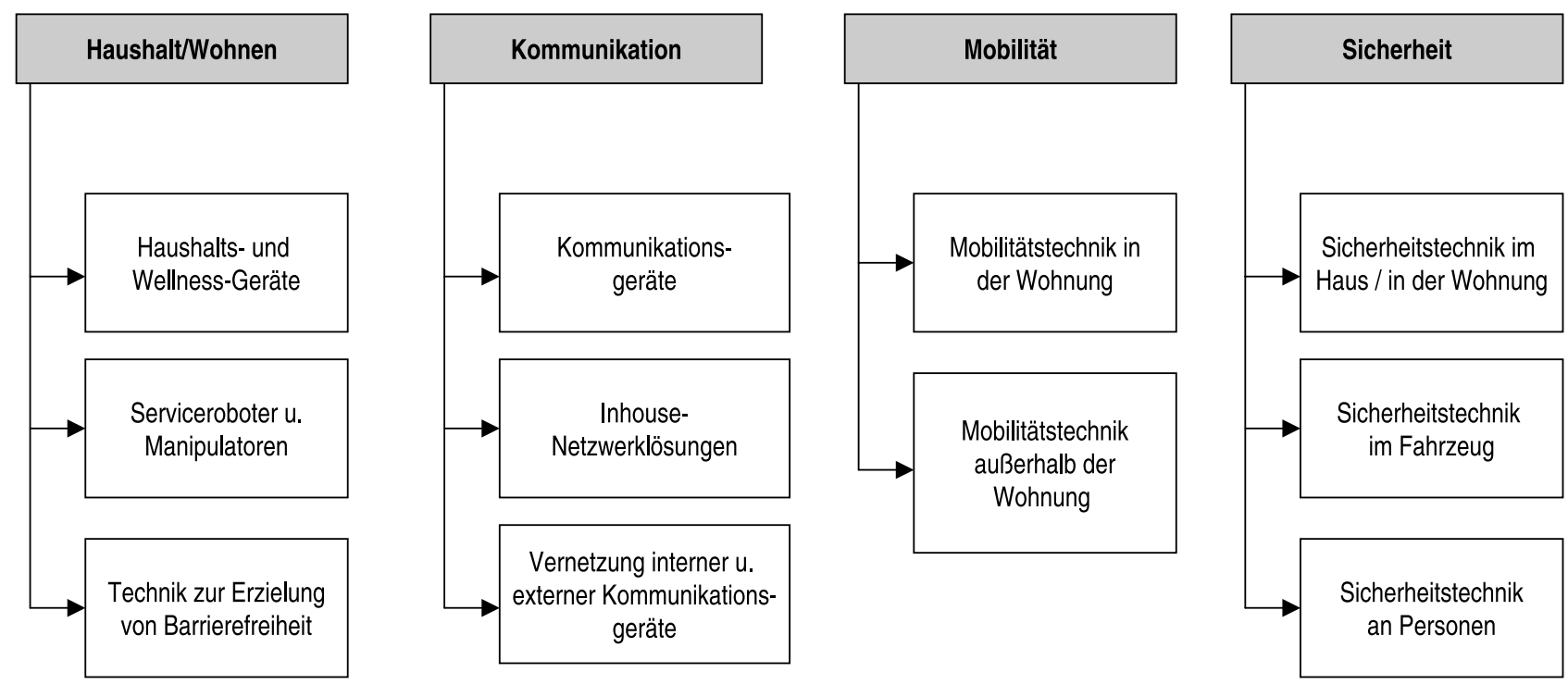

Abb. 1: Produktgruppen Seniorengerechter Technik nach Anwendungsbereichen

Anwendungsbereich „Inhaltsstoffe - non-food“ / Kunststoffe

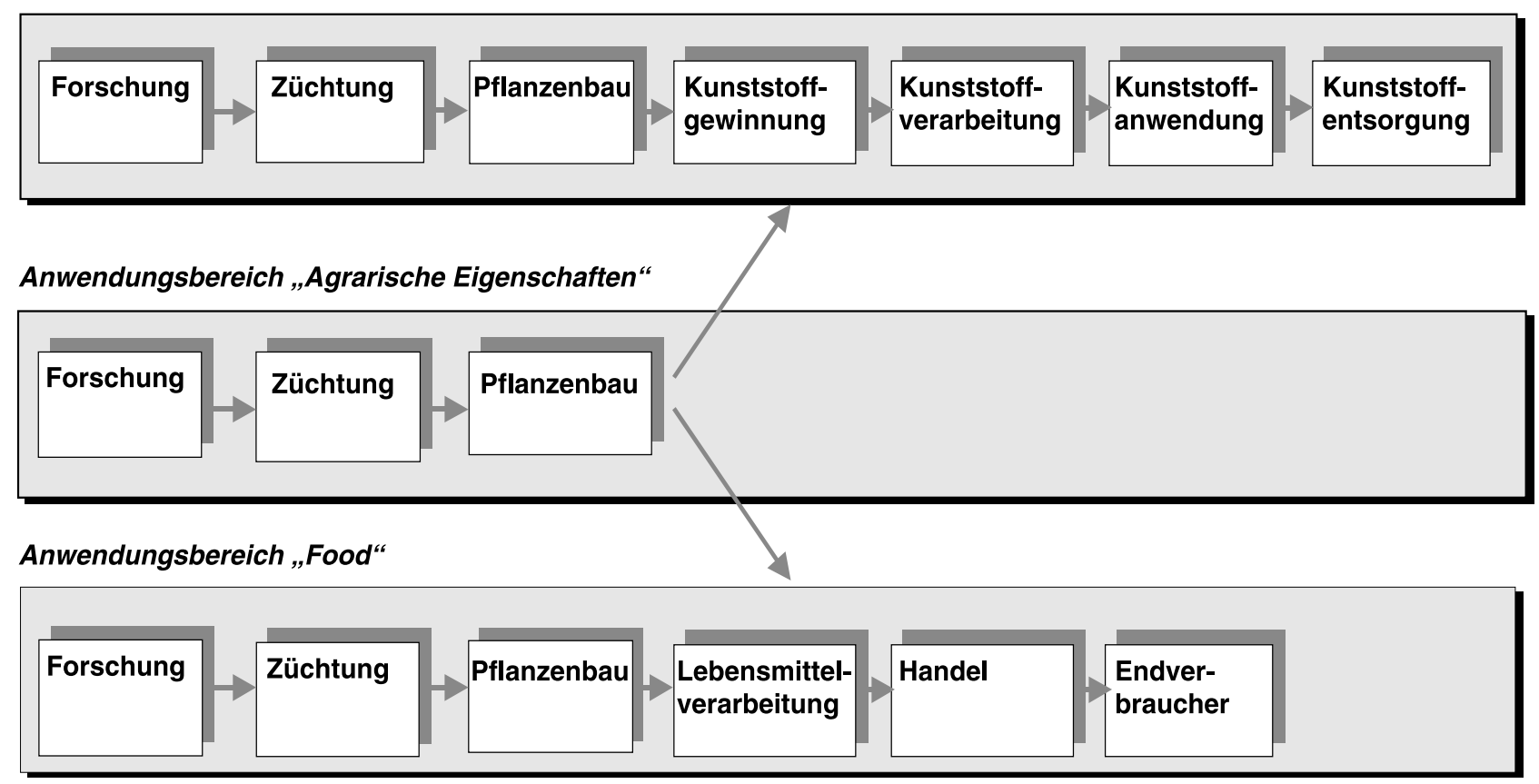

Abb. 2: Wertschöpfungsketten in Anwendungsbereichen der Pflanzengentechnik 
Projekte ihren hohen Differenzierungsgrad über die Untersuchung von unterschiedlichen Anwendungsbereichen. Bei ITA in sehr frühen Phasen von Innovationsprozessen kommt hinzu, dass die Antizipation möglicher Folgen ganz entscheidend von der Analyse jener Anwendungskontexte abhängig ist, in die Innovationen eingebettet werden müssen.

Mit dem genutzten Wertschöpfungskettenansatz rücken potenzielle und aktuelle Nachfrager ins Blickfeld der Untersuchung, jedoch mit unterschiedlicher Schwerpunktsetzung. Während im Pflanzengentechnik-Projekt neben privaten Endverbrauchern auch Unternehmen aus verschiedenen Wirtschaftsbereichen als Verwender im Fokus der Untersuchung stehen, betrifft dies im Senioren-Projekt insbesondere den Senior als privaten Endverbraucher (neben helfenden Angehörigen und professionellen Dienstleistern). Es wird unterstellt, dass dessen Nachfrageverhalten bezogen auf seniorengerechte Technik einerseits durch altersbedingte Veränderungen in Verhaltensvariablen beeinflusst wird. Andererseits beeinflusst auch das Vorhandensein anspruchsgerechter Leistungsangebote das Nachfrageverhalten (vgl. Abbildung 3).
Den Begriff des Anspruchs nutzen beide Projekte, und zwar im Sinne gegenstandsgerichteter Wünsche, die nahe an der Verhaltensoberfläche liegen und eine Chance haben, marktrelevant zu werden. [4] Da sich die jeweils untersuchten Innovationsprozesse jedoch in unterschiedlichen Phasen befinden (mit Ausnahme der Robotik liegen diese Prozesse im Bereich der seniorengerechten Technik nicht in so frühen Phasen wie bei der Pflanzengentechnik), erfolgt dies mit verschiedener Intention. Im Pflanzengentechnik-Projekt vermitteln Ansprüche den Zusammenhang zwischen einer Problemlage, auf die ein Innovationsvorhaben mit seinen potenziellen Folgen in seinen frühen Phasen im hohen Maße orientiert ist, und einer Nachfrage, auf die sich die Leistungsangebote als Ergebnisse von Innovationsprozessen beziehen. Nur wenn Innovationsprozesse und deren Folgen passfähig zu bestimmten Ansprüchen potenzieller Kunden gemacht werden, können sie sich realisieren und es entsteht Nachfrage (vgl. Abbildung 4).

Ansprüiche sind kontextabhängig, historisch gewachsen, aber auch - in unterschiedlichem Maße - veränderlich. Sie werden indirekt $\mathrm{z}$. B. durch politisch-rechtliche und

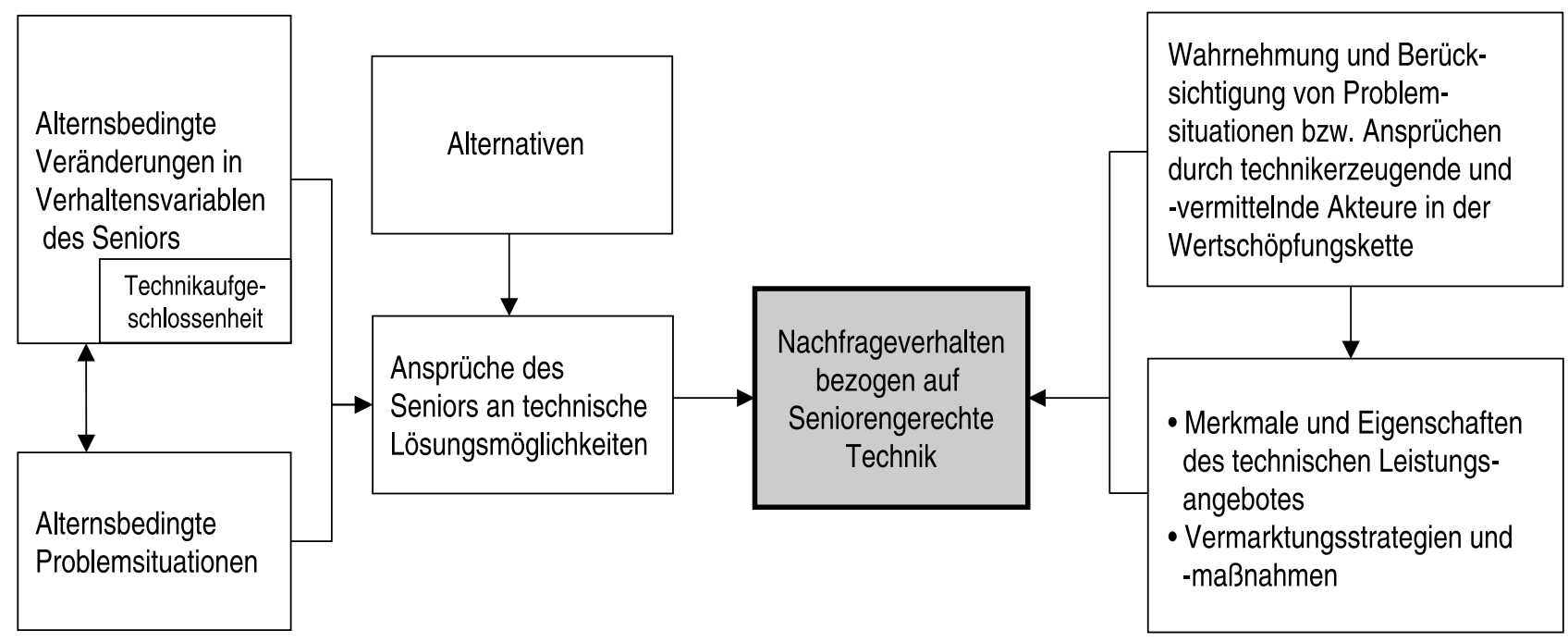

Abb. 3: Schematische Darstellung des Untersuchungskonzepts im Senioren-Projekt

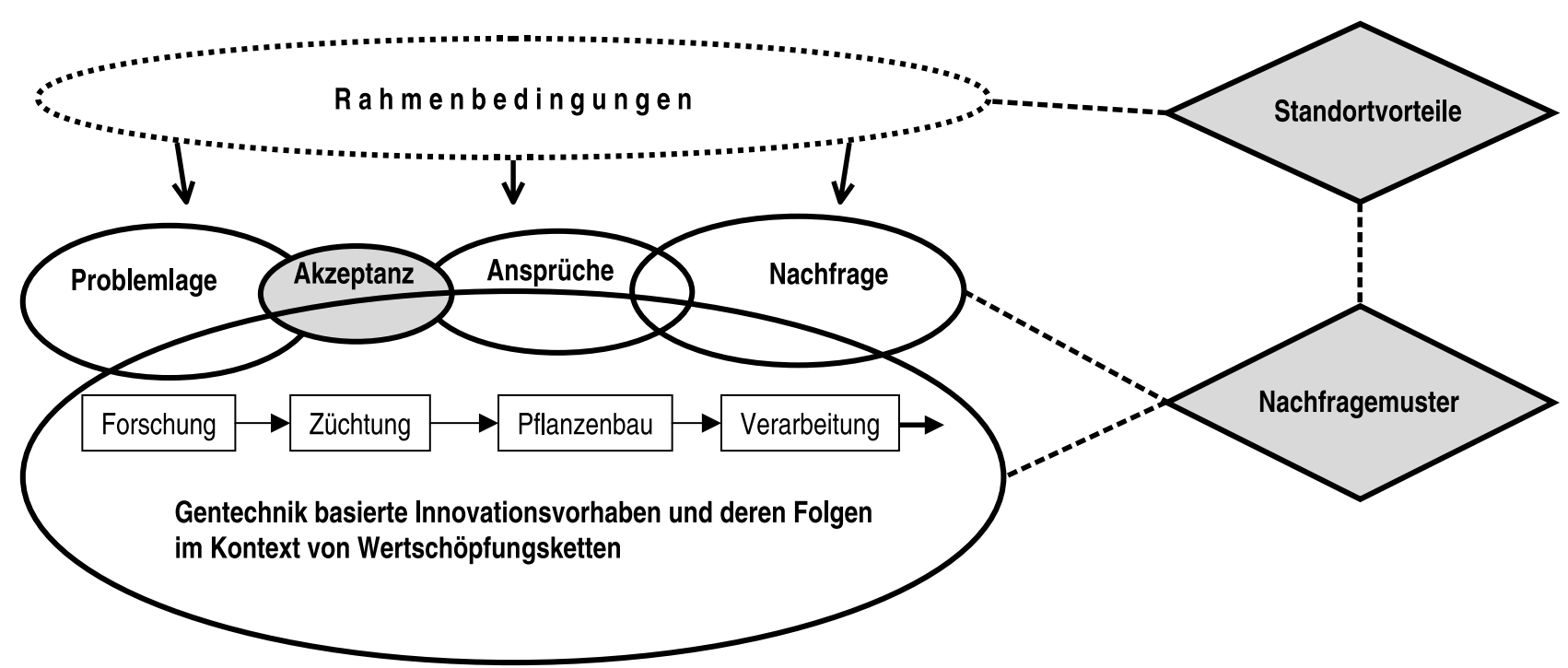

Abb. 4: Schematische Darstellung zum Untersuchungskonzept im Pflanzengentechnik-Projekt 
wirtschaftliche Rahmenbedingungen beeinflusst. Unter Rückgriff auf das Wertschöpfungskettenmodell sind verschiedene Akteure mit Ansprüichen identifizierbar. Hervorgehoben werden im Pflanzengentechnik-Projekt etwa produktive Verwender, Akteure aus dem Bereich Logistik/Handel und private Endverbraucher. Im Senioren-Projekt werden Ansprüche an technische Produkte als aggregierter Ausdruck konkreter Ausprägungen von Verhaltensvariablen bei Senioren genutzt. Für die Herausbildung von Ansprüchen spielen jeweils die verfügbaren Alternativen eine wichtige Rolle. Im Pflanzengentechnik-Projekt sind dies nicht gentechnisch basierte Produkte, im Senioren-Projekt durch Personen erbrachte Leistungen anstelle technischer Leistungsangebote.

Beide Projekte haben sich auf jeweils spezifische Weise mit der Rolle von Einstellungen und dem Begriff der Akzeptanz beschäftigt. Im Senioren-Projekt konnte davon ausgegangen werden, dass auftretende Akzeptanzprobleme in Bezug auf seniorengerechte Technik in der Regel nicht auf fehlender Technikakzeptanz beruhen. Daher war insbesondere die Anwendungsakzeptanz bezogen auf konkrete technische Produkte Untersuchungsgegenstand. Im Pflanzengentechnik-Projekt wurde dagegen explizit zwischen Technikakzeptanz und Anwendungsakzeptanz unterschieden und differenziert untersucht, welche Rolle diese beiden Akzeptanzformen bei Akteuren in den Stufen unterschiedlicher Wertschöpfungsketten, einschließlich privater Endverbraucher sowie der Öffentlichkeit, spielen.

Schließlich wurde in beiden Projekten die Wirkung von Akzeptanz und Nachfrage als Standortvorteil entsprechend der Spezifik der untersuchten Technologiebereiche konzipiert und analysiert. Im Pflanzengentechnik-Projekt fokussiert die Untersuchung auf die Realisierungsbedingungen für erfolgreiche pflanzengentechnisch basierte Innovationsvorhaben und die wirtschaftliche wie gesellschaftliche Integration der Pflanzengentechnik insgesamt. Diese Realisierungsbedingungen schließen potenzialseitige Aspekte, nachfrageseitige Aspekte, politisch-rechtliche Rahmenbedingungen und die Akzeptanz der Pflanzengentechnik in der Öffentlichkeit ein. Dieses Verständnis von Standortvorteilen knuipft an dem regionaler Wettbewerbsvorteile von Michael Porter [5] an, erweitert dieses vor allem hinsichtlich rechtlich-politischer Rahmenbedingungen und spezifiziert es zweifach, indem es Besonderheiten einer „Technologie“ in Frühphasen ihrer Entwicklung betrachtet und die Beziehungen zwischen Akteuren aus der Wirtschaft nicht als reine Zulieferbeziehungen, sondern als Verwenderbeziehungen entlang vertikaler Wertschöpfungsketten konzipiert. Im Senioren-Projekt waren dagegen vor allem die Standortbedingungen für Unternehmen in Deutschland auf den verschiedenen Anwendungsfeldern (z. B. Vorhandensein relevanter Forschungsinfrastruktur, Zentren für Gerontotechnologie), ihre Wettbewerbssituation, die Zutrittschancen für neue Unternehmen und der Vergleich von Rahmenbedingungen mit anderen Ländern untersuchungsleitend.
Hauptuntersuchungsmethode waren in beiden Projekten Fallstudien. Im Pflanzengentechnik-Projekt bezogen sich diese auf folgende Innovationsvorhaben:

- „Fusarienresistenter Weizen“ (Nutzung der Pflanzengentechnik zur Verbesserung agrarischer Eigenschaften);

- „Weizen ohne Zöliakietoxizität“ (Nutzung der Pflanzengentechnik im Food-Bereich);

- „Biologisch abbaubare Kunststoffe aus transgenen Pflanzen“ (Nutzung der Pflanzengentechnik im Bereich Industrierohstoffe).

Im Senioren-Projekt wurden mit den Fallstudien zu Elektromobilen, Navigationssystemen für PKW, Seniorencomputern und Heimrobotern bzw. Roboterassistenten Leistungsangebote analysiert, die sich in unterschiedlichen Phasen des Produktlebenszyklus befinden, bezuiglich ihres Innovationsgrades und Technologiebezuges differieren und von Unternehmen verschiedener Größe entwickelt und produziert werden. Die gewonnenen Ergebnisse wurden mit Exkursen zu Großtastentelefonen und Hausnotrufsystemen gestuitzt.

Die Erarbeitung der Fallstudien und Exkurse basierte auf leitfadengestuitzten ein- bis zweistündigen Experteninterviews mit zahlreichen Schluisselakteuren, Literaturund Dokumentenstudien sowie der Teilnahme an Fachveranstaltungen und -messen. Der fuir die Projektbearbeitung angewandte Methodenmix umfasste des Weiteren im Senioren-Projekt drei kleinere schriftliche Befragungen sowie im Pflanzengentechnik-Projekt eine Internetbefragung zur Ermittlung des Einflusses von Folgenwissen über ein konkretes pflanzengentechnisch basiertes Innovationsvorhaben auf die Akzeptanz dieses Vorhabens durch die Öffentlichkeit.

\section{Hauptergebnisse des ITA-Projekts zur Pflanzengentechnik}

Ausgehend von den Fallstudien wurden in mehreren Schritten Aussagen für komplexe Anwendungsbereiche und die Pflanzengentechnik insgesamt abgeleitet. Zunächst erfolgte die Prüfung der Analyseergebnisse für die untersuchten Innovationsvorhaben hinsichtlich ihrer Verallgemeinerbarkeit für das jeweilige Anwendungsfeld. Daran anschließend konnten Nachfragemuster in der Pflanzengentechnik identifiziert und charakterisiert sowie Standortvorteile ermittelt werden. Die Zusammenschau aller Projektergebnisse (Fallstudienwissen, Befunde der Internetbefragung, Nachfragemuster und Standortvorteile) fuihrt zu folgenden Hauptaussagen und Schlussfolgerungen:

- Die Durchsetzungschancen pflanzengentechnisch basierter Innovationsvorhaben hängen ganz besonders davon ab, ob und wie deren mögliche Folgen mit den Verwenderansprïchen von Wirtschaftsakteuren bzw. privaten Endverbrauchern wechselseitig passfähig gemacht werden können. Nur wenn es gelingt, diese Passfähigkeit herzustellen, kann zukünftig Nachfrage entstehen. 
- Zu pflanzengentechnischen Anwendungen bestehende alternative technologische Entwicklungspfade sind bezogen auf die Nachfrage nicht in jedem Falle als ungünstig zu bewerten. Sie beeinflussen z. B. dann nachfragerelevante Anwendungszusammenhänge positiv, wenn sie mit zeitlichem Vorlauf dazu beitragen, dass Rahmenbedingungen förderlich geändert und Marktbarrieren abgesenkt werden.

- Der geringe Stand der kommerziellen Nutzung der Pflanzengentechnik ist insbesondere auf offene wissenschaftliche und technologische Fragen zurückzufuihren, daneben auf die noch unklare Positionierung der Politik, weniger dagegen auf gentechnikkritische Positionen der Öffentlichkeit. Dennoch ist die Akzeptanz der Pflanzengentechnik durch die Öffentlichkeit eine wichtige Bedingung für ihre wirtschaftliche Umsetzung und gesellschaftliche Integration. Die gängige Annahme, die Pflanzengentechnik werde durch die Öffentlichkeit insgesamt oder grundsätzlich abgelehnt, muss jedoch korrigiert werden.

- Bezogen auf die Pflanzengentechnik erfolgte erstmals die Unterscheidung zwischen der allgemeinen (Pflanzengen)-Technikakzeptanz und der Anwendungsakzeptanz bezogen auf Anwendungsbereiche oder konkrete Anwendungsbeispiele. Es konnte belegt werden, dass Technikakzeptanz und Anwendungsakzeptanz oft übereinstimmen, aber für einzelne Anwendungen auch deutlich voneinander abweichen.

- Mit der Internetbefragung wurde gezeigt, dass sich die Akzeptanz eines pflanzengentechnisch basierten Innovationsvorhabens verändern kann, wenn die Befragten über mögliche Folgen dieses Vorhabens informiert werden. Sie räumten einem solchen Folgenwissen einen höheren Stellenwert ein als anderen Informationen.

- Potenzialseitig verfuigt Deutschland uiber die erforderlichen Voraussetzungen für die Entwicklung und Nutzung der Pflanzengentechnik, und zwar in allen Stufen der drei untersuchten Wertschöpfungsketten. Das wechselseitig aufeinander bezogene Agieren der Akteursgruppen aus diesen Wertschöpfungsstufen im Hinblick auf die Realisierung pflanzengentechnisch basierter Innovationsvorhaben ist jedoch unterschiedlich entwickelt. Während die Beziehungen zwischen Forschung und Pflanzenzuichtung bereits sehr intensiv sind, werden mögliche Folgen pflanzengentechnisch basierter Innovationsvorhaben durch Akteure in der Landwirtschaft und in folgenden industriellen Verarbeitungsstufen zwar wahrgenommen, darauf bezogene Verwenderansprïche aber noch zu wenig formuliert und an die Zuichtung und Forschung durchgestellt.

- Wirtschaftsakteure in den untersuchten Wertschöpfungsketten akzeptieren grundsätzlich den Einsatz der Pflanzengentechnik. Da sie aber undifferenziert von einer überwiegenden Ablehnung der Pflanzengentechnik durch die Öffentlichkeit ausgehen, vertreten sie ihre Akzeptanz nicht in jedem Falle auch öffentlich.

- Erstmalig wurden für die Pflanzengentechnik Nachfragemuster ermittelt. Sie zeigen, in welchen nachfragerelevanten Konstellationen sich eine große Zahl pflanzengentechnisch basierter Innovationsvorhaben entwickelt bzw. sich zukünftig entwickeln wird. Unter Nutzung der Muster können Handlungsbedarfe für Rahmensetzer und Innovatoren abgeleitet werden, nachfragebeeinflussende Faktoren förderlicher zu gestalten und notwendige Kommunikationsprozesse rechtzeitig aufzunehmen.

- Den Flaschenhals für die wirtschaftliche Umsetzung des Potenzials der Pflanzengentechnik bildet eine international wettbewerbsfähige Pflanzenzüchtung. Deshalb sollte seitens der Politik der Entwicklung der Zuichtungsbranche - über die Förderung ihrer Forschungspotenziale hinaus - große Aufmerksamkeit gelten.

- Für die strategische Planung neuer sowie die Realisierung laufender pflanzengentechnischer Innovationsvorhaben muss kurzfristig Planungssicherheit geschaffen werden, was eine klare politische Positionierung sowie den Vollzug bestehender Gesetze voraussetzt.

- Anknüpfend an die These von der Notwendigkeit der Normalisierung hypothetischer Risken der Pflanzengentechnik (van den Daele) kommen die Autoren im Ergebnis der Studie zu der zentralen These von der Notwendigkeit der Normalisierung des Nutzens. Sie liegt insbesondere darin begründet, dass sowohl hochgradig gesellschaftlich relevante und komplexe Problemlagen als auch Problemlagen einzelner privater Endverbraucher in den wenigsten Fällen allein durch Pflanzengentechnik lösbar sind. Eine Ausnahme können einzelne Problemlagen bei Wirtschaftsakteuren bilden. Hinzu kommt, dass die Pflanzengentechnik als Methode heute noch so unreif ist, dass problemlösende Ideen oft noch nicht in entsprechende pflanzengentechnisch basierte Produkte umgesetzt werden können. Eine Normalisierung des Nutzens hätte Konsequenzen für den strategischen Umgang mit der Pflanzengentechnik in Forschung, Wirtschaft und Politik ebenso wie fuir die öffentliche Diskussion und die Akzeptanz der Öffentlichkeit.

Die Ergebnisse insgesamt verdeutlichen den Wert von ITA-Wissen für eine erfolgreiche Realisierung pflanzengentechnisch basierter Innovationsvorhaben ebenso wie für deren gesellschaftliche Implementierung.

\section{Hauptergebnisse des ITA-Projekts zur seniorengerechten Technik}

Mit den Fallstudien für technologische Innovationen in den Anwendungsbereichen „Mobilität“, „Sicherheit“, „Kommunikation“ und „Haushalt“ liegen detaillierte Ergebnisse zu den jeweils typischen altersbedingten 
Problemsituationen von Senioren, zur Befriedigung ihrer produktbezogenen und Vermarktungsansprüche durch die vorhandenen technischen Leistungsangebote, zur Entwicklung der Nachfrage und zu den Standortbedingungen der Unternehmen vor. So konnte z. B. für das Anwendungsfeld „Mobilität“ gezeigt werden, dass

- die Mobilität im außerhäuslichen Bereich einen hohen Stellenwert für die Lebensqualität von Senioren bis ins hohe Alter hat,

- neben dem „normalen“ PKW Elektromobile (Scooter, Leicht-KFZ) eine Option für individuelle Mobilität darstellen, die in Deutschland aber überwiegend nur von Behinderten genutzt wird,

- die Angebotspalette bei Elektroscootern nahezu alle produktbezogenen Ansprïche der Kunden abdeckt, jedoch Defizite bezüglich der Vermarktungsanspriiche auszumachen sind,

- die befragten Firmen eine steigende Nachfrage nach Elektromobilen erwarten,

- die deutschen Anbieter von Elektromobilen einem harten Preiskampf mit ausländischen Konkurrenten ausgesetzt sind.

Bezogen auf das Nachfrageverhalten von Senioren wird nachgewiesen, dass

- nachfragerelevante Problemsituationen meist nicht allein durch altersbedingte Veränderungen in personenbezogenen Variablen entstehen, sondern vielmehr erst in Kombination mit weiteren umgebungsbezogenen Variablen,

- auch Veränderungen in umgebungsbezogenen Variablen allein eine Techniknachfrage auslösen können,

- die Beschaffenheit einer einzigen Verhaltensvariablen alle anderen Variablen mit einem förderlichen Einfluss auf die Nachfrage außer Kraft setzen und die Realisierung der Nachfrage über den Kaufakt verhindern kann (z. B. ein zu geringes Einkommen oder die Nichtwahrnehmung der Problemlage),

- je nach entstandener Problemsituation entweder Technik zur Lösung von Aufgaben nachgefragt wird, die bisher ohne Technik absolviert wurden, oder neue Ansprüche an bereits genutzte Technik formuliert werden bzw. Technik nachgefragt wird, die bisherige Technik substituiert,

- ein gleiches Ausmaß an Veränderung in Verhaltensdeterminanten verschieden starke Auswirkungen auf das Nachfrageverhalten haben kann, wobei die Höhe des Problemdrucks und die Alternativen eine wichtige Rolle spielen,

- geschlechtsspezifische Unterschiede in der Technikaufgeschlossenheit nicht fuir alle Technikanwendungen gleichermaßen relevant sind,

- die überwiegende Mehrheit der Senioren eine hohe Technikaufgeschlossenheit hat, nachgewiesene Unterschiede zwischen allgemeiner und bereichsspezifischer Technikeinstellung sowie zwischen Technikeinstellung, Anschaffung und Nutzung aber durchaus für die Bewertung der Nachfrage berücksichtigt werden müssen,

- auftretende Akzeptanzprobleme in Bezug auf seniorengerechte Technik oftmals durch das vielen Produkten anhaftende „Behindertenimage“ verursacht sind.
Zur Charakterisierung der Angebots- und Anbietersituation wird erstens gezeigt, dass Technikentwickler und -hersteller verschiedener Wertschöpfungsketten den Zusammenhang von altersbedingten Veränderungen in Verhaltensvariablen, Problemsituationen und Ansprüchen auf unterschiedliche Weise wahrnehmen und in entsprechende Handlungsstrategien bei der Entwicklung nachfragegerechter Leistungsangebote umsetzen. Hierzu wird dargestellt

- mit welcher Zielsetzung die markt- oder technologiegetriebenen Produktentwicklungen erfolgen,

- welche Möglichkeiten Technikentwickler und -hersteller haben, die Ansprüche von Senioren an geplante Produktverbesserungen und -neuentwicklungen zu erkennen,

- welche Erfahrungen aus unterschiedlichen Formen des Involvements (z. B. Seniorenpanel, usability labs) resultieren,

- welche Anforderungen an die Produktentwicklung aus den Erkenntnissen der Alternsforschung resultieren (,design for all“ versus Variation und Modularität).

Zweitens werden die Leistungsangebote hinsichtlich ihrer Innovationshöhe, des Technologieniveaus und der sich abzeichnenden technologischen Entwicklungstrends charakterisiert. Letztere gehen in folgende drei Richtungen:

- Verbesserung/Erweiterung der Funktionalität traditioneller technischer Geräte,

- Automatisierungslösungen für bislang nicht von Technik erfasste Tätigkeiten und Prozesse mit neuartigen Geräten,

- Vernetzung hausinterner technischer und elektronischer Geräte wie auch Vernetzung hausinterner mit externer Technik.

Drittens werden die Erfahrungen aus unterschiedlichen Vermarktungsstrategien und Vertriebswegen beschrieben und typische Vermarktungsprobleme der mittelständischen Firmen identifiziert. Auf dem Zuwendungsmarkt $^{2}$ können diese z.B. aus dem Verhalten einiger Technikvermittler resultieren. Auf dem Leistungsaustauschmarkt fehlt den kleineren Firmen z.B. der Zugang zu effektiven Vertriebskanälen und aufgrund eingeschränkter Werbemöglichkeiten erreichen ihre Produkte nicht den gewünschten Bekanntheitsgrad.

Es wird gezeigt, dass die gesellschaftliche Aufmerksamkeit für die altersbedingten Problemlagen älterer Menschen in den untersuchten Anwendungsbereichen unterschiedlich stark ausgeprägt und akzentuiert ist. So wird beispielsweise die Nutzung moderner Informations- und Kommunikationstechnologien durch ältere Menschen in Deutschland seit etwa fuinf Jahren spürbar durch Politiker, Forscher, Vereine und Verbände, Unternehmen und nicht zuletzt die Senioren selbst thematisiert. Dagegen wird das Problem der Gewährleistung der individuellen Mobilität für ältere Menschen, die kein Auto nutzen können, öffentlich kaum wahrgenommen. Beispielhaft wird der Einfluss gesetzlicher Regelungen auf die Nachfrage aufgezeigt. So wirkt die Aufnahme der Elektroscooter in das Hilfsmittelverzeichnis der Krankenkassen diesbezüglich positiv, während die bis Anfang vorigen Jahres gültige Regelung, geschlossene einsitzige Kran- 
kenfahrstühle nur für behinderte Personen zuzulassen, einer Nachfrage entgegen stand.

Basierend auf der Analyse der Anbietersituation nach der Anzahl und Größe der Anbieterfirmen, ihrer Herkunft (Brancheninsider oder branchenfremd), der Konkurrenzsituation, der Marktgröße und der Zutrittschancen fuir neue Unternehmen wird bezüglich der Standortbedingungen für Unternehmen belegt, dass nicht in allen Bereichen gute Zutrittschancen für neue Firmen bestehen und diese zum Teil in unterschiedlich begründeten „Zeitfenstern" möglich und sinnvoll sind (Seniorencomputer, Roboterassistent). Als positive Standortbedingungen werden u. a. die spezifischen Zentren für Gerontotechnik bzw. Gerontotechnologie in Iserlohn und Bad Tölz sowie die leistungsfähige Forschungs- und Unternehmenslandschaft auf dem Gebiet der Robotik herausgearbeitet.

Der konzeptionelle Zugang des vorliegenden Projekts und die erarbeiteten Fallstudien haben eine neue Sicht auf vorliegende Ergebnisse der Alternsforschung und der Vermarktung ermöglicht und die Schnittstellen zu deren Nutzung bei der Umsetzung in nachfragegerechte technische Leistungsangebote durch Unternehmen aufgezeigt. Es wurde zugleich herausgearbeitet, dass die Berücksichtigung der Ergebnisse der Alternsforschung und Konsumentenforschung allein nicht alle bei der Umsetzung auftretenden Fragen beantworten kann. Vielmehr muissen dazu auch wirtschafts- und sozialwissenschaftliche Forschungen Beiträge leisten. Es wird insbesondere vorgeschlagen,

- sowohl Instrumente des Innovationsmanagements, der Wettbewerbstheorie und des Marketings, als auch Erkenntnisse der interdisziplinären Alternsforschung (z. B. zum Entwicklungspotenzial, zur Variabilität, Kompetenz und Plastizität und zu den differenzierten Lebensstilen von Senioren) verstärkt für die Technikentwickler und -hersteller handhabbar zu machen und gewissermaßen in Strategien, Ziele und ein entsprechendes Innovationsverhalten bezogen auf kundengerechte Leistungsangebote für ältere Menschen zu transformieren,

- neue theoretische und empirisch überpriffbare Konzepte, z. B. zur Rolle von Technik bei der Förderung und Erhaltung von Kompetenzen, zu schaffen. Diese sollten interdisziplinär unter Beteiligung von Experten verschiedenster Wissenschaftsdisziplinen (Geriatrie, Medizin, Psychologie, Soziologie, ...) sowie von Forschern und Entwicklern aus den jeweiligen Technikbereichen erarbeitet werden,

- dass alle an einem zufriedenen und erfolgreichen Altern zukünftiger Seniorengenerationen interessierten Akteure (Senioren, Mediziner, Alternsforscher, Politiker, Technikentwickler und -hersteller, Dienstleister, ...) in diskursiven Prozessen begründete Szenarien zu langfristig sinnvollen Strategien für den Umgang mit individuellen und gesellschaftlichen Alterungsprozessen und der Rolle, die Technik dabei spielen sollte, erarbeiten und daraus Maßnahmen zu ihrer praktikablen Handhabung in der Alternsforschung, in Wirtschaft und Politik ableiten.

\section{Erfahrungen aus der Projektbearbeitung}

Die Operationalisierung des in wesentlichen Aspekten im Kapitel 2 beschriebenen Untersuchungskonzeptes und dessen Anwendung in den beiden Projekten fuihrte zu einer Reihe von Umsetzungserfahrungen, die im Folgenden kurz dargestellt werden.

Es konnte gezeigt werden, dass das ITA-Konzept der Autoren auf ganz unterschiedliche Technologiebereiche und Untersuchungsbeispiele anwendbar ist. Seine Anwendung fuihrt zu differenzierten, wohl begründeten Aussagen auf unterschiedlichste Fragestellungen im Rahmen von ITA.

Unternehmen als eine wichtige Zielgruppe für Innovations- und Technikanalysen für derartige Untersuchungen zu interessieren, ist nicht einfach. Dies belegen bereits die Erfahrungen mit den klassischen TA-Studien der Vergangenheit. [6] Mit den beschriebenen Projekten wurde ein Weg beschritten, Unternehmen nicht nur für die analysierten Fragestellungen zu interessieren, sondern ihre Erfahrungen unmittelbar für die Projektbearbeitung zu nutzen. Dies gelang insbesondere durch

- die thematische Fokussierung der Projekte auf nachfragerelevante Situationen, Bedingungen und Faktoren, was für Unternehmen naturgemäß von unmittelbarem Interesse ist,

- den gewählten konzeptionellen Zugang über konkrete Innovationslinien und Wertschöpfungsketten, durch den für die Unternehmen das forschungsseitige Interesse an ihren Leistungserstellungsprozessen klar erkennbar wurde,

- die Einbeziehung von Standortfaktoren und ausgewählten gesellschaftlichen Rahmenbedingungen für mittelständische und große Unternehmen in den untersuchten Anwendungsbereichen,

- die Herausarbeitung wichtiger, uiber die Wertschöpfungsketten vermittelter, Beziehungen zwischen den Unternehmen sowie zwischen Wirtschaft und Wissenschaft und

- die Durchfuihrung von Expertengesprächen mit nahezu sämtlichen Schlüsselakteuren der untersuchten Wertschöpfungsketten.

Die interviewten Experten aus den Unternehmen und der wirtschaftsnahen Forschung zeigten sich den bearbeiteten Themen gegenuiber sehr aufgeschlossen und waren bereit, sich sowohl in sehr zeitintensiven Gesprächen kreativ in die Projektbearbeitung einzubringen, als auch im Nachhinein die jeweils erstellten Fallstudientexte zu lesen und kritisch zu prüfen. Von den Bearbeitern erfordert die Umsetzung des Konzeptes, sich für jede Fallstudie eine komplexe Wissensbasis zu Befunden verschiedenster Wissenschaftsdisziplinen und deren mannigfaltigen Zusammenhängen zu erarbeiten. Diese Wissensbasis ist eine wesentliche Voraussetzung dafür, die in den ITA-Projekten zu klärenden Fragen herauszuarbeiten und Expertenwissen erschließen zu können.

In frühen Phasen von Innovationsprozessen ist die Antizipation ein geeignetes Mittel, um vor dem Hinter- 
grund von bereits gesichertem Wissen über Anwendungskontexte deren Entwicklung sowie die Folgen von Innovationsprozessen begrüindet abzuschätzen.

Die konzeptionelle Verortung des Untersuchungsgegenstandes Akzeptanz und Nachfrage als Standortvorteil im Gebiet ITA erforderte die Auswertung und Integration verschiedener wissenschaftlicher Ansätze. Dazu gehören insbesondere Konzepte aus der sozialwissenschaftlichen Forschung, z. B. der Innovationsforschung, der Technikgeneseforschung und der politikwissenschaftlichen Forschung sowie aus der Betriebswirtschaft, z. B. zum Marketing und Konsumentenverhalten.

Die konsequente Umsetzung des entwickelten Konzeptes war nur durch eine differenzierte Untersuchung mit tiefgehenden Fallstudien möglich. Dies erforderte die Aneignung zahlreicher wissenschaftlicher Erkenntnisse aus weiteren Wissenschaftsbereichen. Im Senioren-Projekt betraf dies z. B. die verschiedenen Ansätze und Ergebnisse der psychologischen und medizinischen Alternsforschung zu typischen individuellen und gesellschaftlichen Alternsprozessen fuir gegenwärtige und auch künftige Seniorengenerationen sowie aktuelle Ergebnisse der ingenieurwissenschaftlichen Forschung aus den Bereichen Mobilitäts-, Sicherheits- und Kommunikationstechnik sowie Robotik. Im Pflanzengentechnik-Projekt wurden insbesondere Ergebnisse aus der Ernährungsforschung, der Medizin, der Ökologie, Ökotoxikologie, Pflanzenzüichtung, Kunststoffforschung und Lebensmitteltechnologie recherchiert, bewertet und verknuipft.

Durch die Auseinandersetzung mit unterschiedlichen Formen der Nutzerintegration im Prozess der Leistungserstellung seniorengerechter Technik konnte dem ITAAnspruch, den Partizipationsgedanken auch auf unmittelbar unternehmensrelevante Fragestellungen auszudehnen, Rechnung getragen werden. Dabei gelang eine differenzierende Sicht dergestalt, dass die Sinnfälligkeit einer Nutzerintegration bezüglich Umfang, Art und Weise sowie Zeitpunkt fallspezifisch zu bewerten ist und keinesfalls als Garant für die gewünschte Nachfrage angesehen werden darf.

Die Ergebnisse beider Projekte bestätigen nicht nur die Notwendigkeit hochgradig differenzierter, ggf. auch fallbezogener Innovations- und Technikanalysen mit erheblicher Untersuchungstiefe. So kann das Risiko von Fehleinschätzungen, z. B. bezüglich der Nachfrage, verringert werden. Sie zeigen auch den Bedarf an geeigneten Methoden, um von solchen Ergebnissen zu verallgemeinerbaren Aussagen zu gelangen - ein Feld, in dem noch Forschungsarbeit zu leisten ist.

\section{Anmerkungen}

1 Beide Projektberichte stellt der Projektträger unter www. innovationsanalysen.de als Download zur Verfuigung.

2 Im Gegensatz zum freien Leistungsaustauschmarkt handelt es sich beim Zuwendungsmarkt um Produkte, deren Finanzierung ganz oder teilweise durch Kranken- oder Pflegekassen erfolgt.

\section{Quellen}

[1] vgl. BMBF (Hrsg.): Innovations- und Technikanalyse: Zukunftschancen erkennen und realisieren. Bonn 2001, S. 23

[2] www.innovationsanalysen.de

[3] vgl. Kowol, Uli: Innovationsnetzwerke. Technikentwicklung zwischen Nutzungsvisionen und Verwendungspraxis. Wiesbaden 1998, S. 361

[4] vgl. Koppelmann, Udo: Produktmarketing. Berlin/Heidelberg 2001, S. 136

[5] vgl. Porter, Michael, E.: On Competition. Boston 1998, p. 211

[6] Weber, Jürgen; Schäfer, Utz; Hoffmann, Dirk; Kehrmann, Titus: Technology Assessment. Wiesbaden 1999, S. 256

\section{Autoren}

Dr. oec. Martina Brandt

Technische Fachhochschule Wildau

Fachbereich BW/WI

Forschungsgruppe „Regionale Innovationscluster“

Telefon +493375 508-512

E-Mail: mbrandt@wi-bw.tfh-wildau.de

Dr. rer. nat. Ulla Große

Technische Fachhochschule Wildau

Fachbereich BW/WI

Forschungsgruppe „Regionale Innovationscluster“

Telefon +493375 508-512

E-Mail: ugrosse@wi-bw.tfh-wildau.de

Dr. phil. Frank Hartmann

Technische Fachhochschule Wildau

Fachbereich BW/WI

Forschungsgruppe „Regionale Innovationscluster“

Telefon: +49 3375 508-214

E-Mail: fhartman@wi-bw.tfh-wildau.de

\section{Prof. Dr. phil. Rainer Voß}

Technische Fachhochschule Wildau

Dekan des Fachbereichs BW/WI

Leiter der Forschungsgruppe „Regionale Innovationscluster" und Direktor des Instituts für Regionale Innovationsforschung Berlin/Wildau

Telefon: +493375 508-951

E-Mail: rvoss@igw.tfh-wildau.de 